Investigación Experimental

Pensar en Movimiento:

Revista de Ciencias del Ejercicio y la Salud

EISSN 1659-4436

Vol. 11, No.2, pp. 1- 17

\title{
BÚSQUEDA DE TALENTOS DEPORTIVOS EN HALTEROFILIA: VALIDACIÓN DE TEST
}

\author{
Jimmy Fernando Rojas Quirós, Lic \\ jiroqui@gmail.com \\ Estudiante del programa de Maestría en Salud Integral y Movimiento Humano, \\ Universidad Nacional, Costa Rica
}

Manuscrito recibido: 01/03/2013; reenvíos: 17/06/2013, 11/08/2013, 28/08/2013; aceptado: 04/09/2013;

publicado: 06/11/2013

\section{RESUMEN}

Rojas-Quirós, J.F. (2013). Búsqueda de talentos deportivo en halterofilia: Validación de Test. Pensar en Movimiento: Revista de Ciencias del Ejercicio y la Salud, 11 (2), 1-17. El propósito del estudio fue validar una modificación al test de lanzamiento del balón medicinal de $2 \mathrm{~kg}$, que facilite la búsqueda de talentos deportivos en halterofilia en edades infantiles, se evaluó la fuerza explosiva a través de un gesto técnico similar al levantamiento del arranque. Participaron 65 niños, $47.7 \%$ hombres y $52.3 \%$ mujeres, de una edad promedio de 10.88 (.72) años. Después de un calentamiento, efectuaron un test modificado de lanzamiento del balón medicinal de $2 \mathrm{~kg}$ y en la misma sesión de trabajo realizaron una prueba ya validada de lanzamiento del balón medicinal de $2 \mathrm{~kg}$. Esto para obtener la validez del test modificado; para finalizar la aplicación de las mediciones se repitió la prueba modificada con el fin de obtener la confiabilidad de la misma. Se ejecutó un análisis correlacional de Pearson ( $r$ ) para obtener tanto la validez como la confiabilidad (test-retest); se utilizó el programa estadístico SPSS versión 18.0 con un nivel de significancia de .05. La correlación entre el Test Modificado y el Test validado de Lanzamiento del Balón Medicinal es de $r=.533 p<.001$; en el análisis de test retest la correlación es de $r=.454 \mathrm{p}=.007$. En conclusión, sí existe una correlación directa entre el test modificado y el test validado de lanzamiento del balón medicinal, por lo tanto el test analizado es válido y a su vez es confiable (test-retest); de igual forma cumple con estas características de validez y confiabilidad para ambos sexos en el análisis según el género.

Palabras clave: Halterofilia, balón medicinal, test, validación, técnica de arranque 


\section{ABSTRACT}

Rojas-Quirós, J.F. (2013). Sports Talent Identification in Weightlifting: Test Validation. PenSAR EN MOVIMIENTO: Revista de Ciencias del Ejercicio y la Salud, 11 (2), 1-17. The purpose of the study was to validate a modification to the $2 \mathrm{~kg}$ medicine ball throw test, which facilitates the search for talented school- age children for Olympic weightlifting. The explosive strength was assessed through a technical movement similar to the weightlifting snatch. 65 children participated, $47.7 \%$ male and $52.3 \%$ female, the average age was 10.88 (.72) years. After a warm up, the modified $2 \mathrm{~kg}$ medicine ball throw test was conducted and in the same work session a previously validated $2 \mathrm{~kg}$ medicine ball throw test was performed too. This was done to validate the modified test. To finish the process the modified test was repeated to verify its test-retest reliability. The Pearson correlation analysis was performed to validate and determine validity and reliability of both the test and re-test. The SPSS version 18.0 was used with a significance level of .05. The correlation between the Modified Test and the validated $2 \mathrm{~kg}$ medicine ball throw test was $r=.533, p<.001$, in the analysis of test - retest, the correlation was $r=.454, p=.007$. Therefore, there is a moderate direct correlation between the test and the validated $2 \mathrm{~kg}$ medicine ball throw test. The analysis shows this test is valid and reliable, and meets the characteristics of validity and reliability for both sexes in the analysis by gender.

Key words: Physical education; weightlifting, sports talent, test,

La halterofilia o levantamiento de pesas es un deporte olímpico donde se compite en dos modalidades o ejercicios, arranque y envión; la ejecución de estas modalidades es una combinación entre técnica y fuerza explosiva del levantador; sin embargo el arranque requiere de una mayor preparación técnica, pues su ejecución consiste en levantar la palanqueta desde la plataforma hasta encima de la cabeza en un solo movimiento en sentido contrario a la gravedad (Ortiz, Navarro, Poletaev y Rausell, 1999; Galván, 2008; Frölich et al., 2005).

La ejecución de una técnica correcta es uno de los principales factores que influyen en el rendimiento en este deporte, pero, también es necesario poseer una gran cantidad de fibras rápidas, para la producción de fuerza explosiva en las piernas y en el tronco, así como una buena flexibilidad en hombros, codos, caderas y tobillos (Ortiz et al., 1999); querer acelerar el proceso de ganancia en el rendimiento o tratar de imponérselo a personas que no son aptos para estas exigencias, provocará como consecuencia una limitación para alcanzar un adecuado rendimiento deportivo.

En los planteamientos tradicionales de la selección de talentos deportivos se ha propuesto establecer las condiciones de base propicias para que un gran número de individuos practicasen deporte organizadamente y tuvieran acceso a la participación controlada en la competición oficial. Dadas dichas condiciones, por un proceso de decantación y selección natural, se irían eliminando todos aquellos que no pudieran tener la progresión necesaria para acceder a cotas sucesivamente más altas del rendimiento deportivo; y como resultado de dicho proceso de eliminación-selección progresiva, los sobrevivientes que alcanzaran la cúspide de la pirámide representarían el nivel de la élite del sistema (Sánchez, 2003); sin embargo, este es un proceso muy largo y costoso en cualquier modelo de desarrollo deportivo, por ello la 
importancia de contar con herramientas de evaluación que permitan detectar con prontitud a los talentos y así minimizar la inversión con un alto porcentaje de efectividad.

El entrenador tiene el deber de ayudar al deportista a desarrollar su talento al máximo de su potencial, esto incluye no solo el desarrollo de los atributos físicos, sino en aspectos como la actitud, los valores, la personalidad, la motivación o autoestima, entre otros (Amebar y Arruza et al., 2002).

Ahora bien, el tipo de test que se aplique debe ajustarse a los conocimientos científicos que se refieren al entrenamiento de la fuerza en niños, no se debe comenzar la preparación sistemática de la fuerza explosiva sin un entrenamiento previo de la fuerza dinámica y de la fuerza estática; por ello los saltos, los lanzamientos variados con balones medicinales o el golpeo sobre móviles tienen una menor posibilidad de producir efectos negativos sobre el aparato locomotor que aun un ejercicio de sentadilla con una ejecución técnica correcta y carga adecuada a las posibilidades del niño (Pastor, 2007).

Durante muchos años el tema de la fuerza en niños ha sido muy reservado y muy pocos han creído que un entrenamiento contra resistencias pueda mejorar la fuerza de los niños; los entrenadores y profesores deben comprender que los infantes pueden beneficiarse de un programa de entrenamiento de la fuerza correctamente planteado y adecuado con su edad (Frölich et al., 2005); es por ello que no se debe utilizar nunca pruebas evaluativas (test) al $1 \mathrm{RM}$, se debe tener en cuenta que ni siquiera son recomendables en deportistas adultos y con experiencia en el entrenamiento de fuerza (Pastor, 2007); para esto se deben buscar otras alternativas de evaluación.

\section{Metodología}

Participantes. La muestra seleccionada consiste en estudiantes de primaria de los niveles de quinto y sexto grado del II Ciclo de la educación general básica. La selección de la muestra se da por conveniencia; participaron tanto hombres como mujeres de ambos niveles.

Instrumentos y materiales. Los instrumentos utilizados se seleccionaron por sus características prácticas para la utilización en el campo, entre ellas: que tenga aplicabilidad, que sus elementos sean sencillos, diseñados para un espacio razonable para su ejecución y sobre todo altos porcentajes de validez y confiabilidad. Se utilizaron dos tests de campo o pruebas de ejecución, a saber:

- Test de lanzamiento de balón medicinal descrito por Ortiz et al. (1999) y Martínez (2002), el cual tiene como objetivo medir la fuerza explosiva sobre todo del tren superior. La confiabilidad obtenida por Guio (2007) a través de un test - retest es de $r=.98$; mientras que Alba (1996) reporta un coeficiente de correlación de $r=.96$. Márquez y Fernández (2012) reportan una validez del test según el coeficiente de correlación intraclase (CCl) de .98, entre las medias de cada modalidad de lanzamiento (de pie, de rodillas, sentado, con una mano); y un coeficiente de correlación de $r=.49, p<.01$.

- Por otra parte, se utilizó una versión modificada de este test, misma utilizada ya hace tiempo por los entrenadores de halterofilia y presentada por el Aján y Baroga (1988). Es de interés de este estudio el validarlo y obtener su confiabilidad en poblaciones 
infantiles; este test pretende medir la fuerza explosiva de tren inferior, específicamente del cuádriceps; así como del tren superior, los músculos de la parte baja de la espalda, el deltoides y el dorsal ancho. El objetivo es utilizar el test como una herramienta en la búsqueda de talentos deportivos en la halterofilia y/o deportes con características biomecánicas similares. En la halterofilia, específicamente, se ejecuta un gesto técnico similar a la técnica de arranque.

Para el desarrollo de ambos test se realizó en un espacio abierto y se necesitaron dos balones medicinales, uno de $1 \mathrm{~kg}$ y otro de $2 \mathrm{~kg}$, una cinta métrica en centímetros, conos y las hojas de recolección de datos.

\section{Procedimientos}

Calentamiento. Para la ejecución de ambos protocolos se procedió a realizar con los participantes un calentamiento, mismo que fue dirigido por el aplicador de la prueba, se realizó en primera instancia una fase breve de carrera continua, seguida de una fase de movilidad articular y trabajos de estiramientos. Seguido a esta rutina se estableció una fase de familiarización, en la cual cada participante practicó la técnica de ejecución con un balón medicinal de $1 \mathrm{~kg}$, cada ejercicio se ejecutó en 2 series de 2 repeticiones.

\section{Test de Lanzamiento del balón medicinal}

Posición Inicial: La persona se coloca en posición de pie, detrás de la línea de lanzamiento, con los pies separados a lo ancho de los hombros. El cuerpo está dispuesto en dirección al lanzamiento y tendrá el balón agarrado con ambas manos de forma simétrica.

Ejecución: A la señal la o el ejecutante eleva el balón medicinal con ambas manos por encima y detrás de la cabeza. Simultáneamente podrá extender el tronco, flexionar brazos y piernas, elevando talones, pero sin despegar las puntas de los pies. A partir de aquí realizará un movimiento explosivo de lanzamiento hacia adelante, con el objetivo de lograr la mayor distancia posible.

El registro de la distancia alcanzada se mide desde la línea de lanzamiento hasta el punto de primer contacto del balón con el piso. Cada participante tuvo oportunidad de efectuar tres intentos con un descanso de 30 segundos, entre estos intentos, se registró la mayor marca realizada por los tres. Durante los intentos se le informaba al participante sus resultados y se le motivaba a mejorarlos en el siguiente.

\section{Test Modificado de Lanzamiento del balón medicinal}

Posición Inicial: La persona se coloca en posición de pie, detrás de la línea de lanzamiento, con los pies separados a lo ancho de los hombros. El cuerpo está de espaldas a la dirección al lanzamiento y tendrá el balón agarrado con ambas manos de forma simétrica.

Ejecución: A la señal los ejecutantes flexionan sus rodillas hasta un ángulo de $90^{\circ}$, los brazos están colgando en posición de péndulo, espalda recta, vista al frente. A partir de aquí realizará un movimiento explosivo de lanzamiento, para ello eleva el balón medicinal con ambas manos por encima y hacia atrás de la cabeza, simultáneamente podrá extender el tronco, extender los brazos y piernas, elevando talones, se puede despegar las puntas de los pies, con el objetivo de lograr la mayor distancia posible. 
El lanzamiento se mide desde la línea de lanzamiento hasta el punto de primer contacto del balón con el suelo, y se registra la distancia alcanzada (Figura 1).

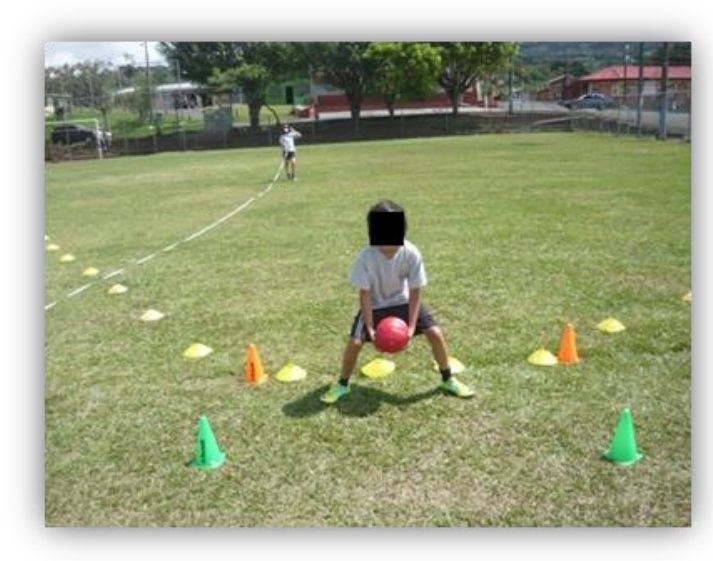

Posición inicial, plano frontal

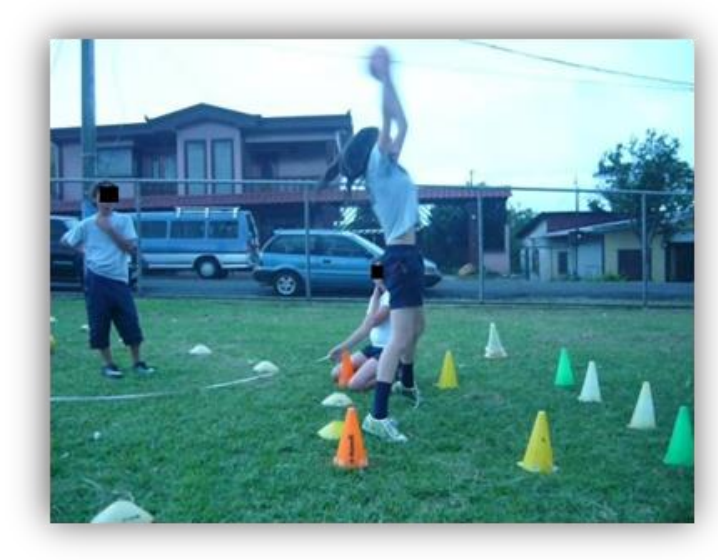

Posición final, plano sagital

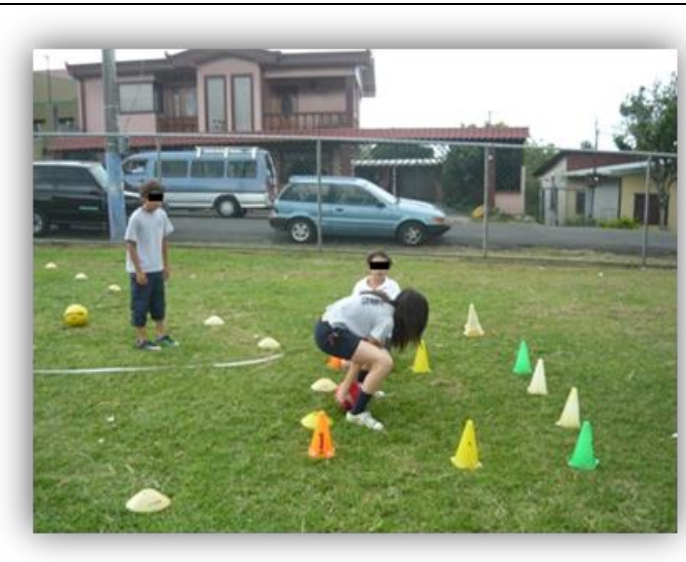

Posición inicial, plano sagital

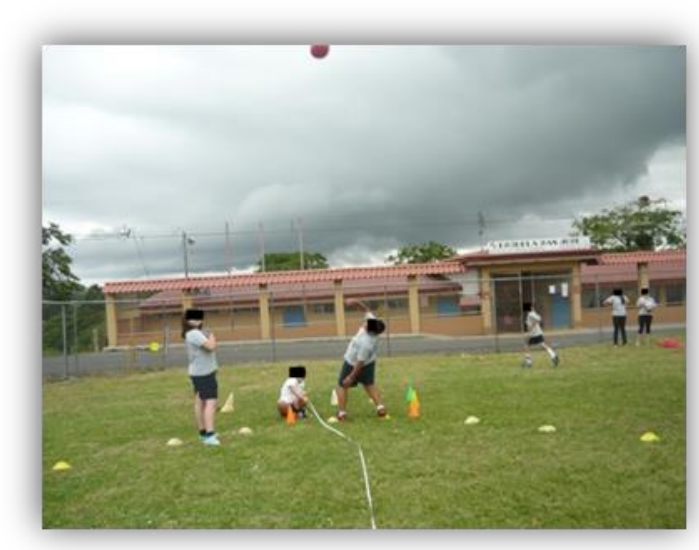

Posición final, plano frontal (atrás)

Figura 1. Técnica de ejecución del Test Modificado de Lanzamiento del Balón Medicinal de 2 $\mathrm{kg}$. En las fotografías participantes del test en plena ejecución del mismo en diferentes fases del movimiento.

De igual forma, cada participante tuvo oportunidad de efectuar tres intentos con un descanso de 30 segundos entre estos intentos y se registró la mayor marca realizada por los tres. Durante los intentos se le informaba al participante sus resultados y se le motivaba a mejorarlos en el siguiente intento.

Análisis Estadístico. Asimismo, se obtuvo la estadística descriptiva (medias, desviaciones estándar) de cada una de las variables. Para conseguir la validez del test 
modificado, se procedió a efectuar un análisis estadístico de correlación de Pearson (r); en primera instancia se hizo una correlación de los datos obtenidos del nuevo test, con los datos obtenidos de la misma muestra del test de lanzamiento del balón medicinal, el cual se encuentra validado para medir la fuerza explosiva del tren superior.

Seguidamente, se continuó con el mismo procedimiento para verificar si la validez aplica indiferentemente del género, para ello se efectuó para ambos sexos análisis estadísticos de Pearson ( $r$ ) para obtener los coeficientes de correlación y su respectiva validación.

La confiabilidad del test se obtuvo a través del protocolo de test - retest, los resultados se analizaron a través de una correlación de Pearson, para ello se correlacionó los datos de la primera medición del test modificado de lanzamiento del balón medicinal, con la segunda medición del mismo test. De igual forma se realizó el procedimiento de confiabilidad para hombres y mujeres. Todos los análisis fueron realizados con el software SPSS versión 18.0 y el nivel de significancia fue de .05 .

\section{Resultados}

La muestra se compone de 65 participantes pertenecientes a los niveles de quinto y sexto grado del II Ciclo de la educación general básica. El promedio de edad de la muestra fue de 10.88 (.718) años, los mayores de los participantes tenían 12 años y los menores 10 años. Un $52.3 \%$ de mujeres, mientras que un $47.7 \%$ de varones.

Por lo tanto, se procedió a la validación del Test Modificado de Lanzamiento del Balón Medicinal, para ello se correlacionó con el Test de Lanzamiento del Balón medicinal que se encuentra validado, en la Tabla 1 se muestra la estadística descriptiva en la relación entre el nuevo test y el test ya validado; y en la figura 2 la correlación entre ambas variables.

\section{Tabla 1}

Estadística descriptiva en la relación entre el Test Modificado de Lanzamiento del Balón Medicinal y el Test validado de Lanzamiento del Balón Medicinal, en estudiantes de primaria

\begin{tabular}{cc}
\hline Variable & M \\
\hline Test Modificado & $409.51(140.02)$ \\
Test Validado & $353.51(86.27)$ \\
\hline
\end{tabular}

Nota: se presentan valores promedio (desviaciones estándar) de las distancias obtenidas en cada una de las pruebas, los datos se encuentran en centímetros $(\mathrm{cm})$. La cantidad de participantes fue de 65.

Al realizar el análisis estadístico de correlación de Pearson, se observa una correlación directa entre el test modificado de lanzamiento del balón medicinal y el test validado de lanzamiento del balón medicinal $(r=.533 ; p<.001)$; por ende a mayor distancia obtenida en el 
test modificado, mayor distancia obtendrá en el test validado; para Martínez (2002) el coeficiente de correlación $(r=.533)$ representa una "relación baja o dudosa" (p. 37); para McMillan y Schumacher (2005) el coeficiente de correlación $(r=.533)$ muestra una "relación positiva moderada" (p. 207); para ambos autores la correlación existe pero no es tan fuerte, esto se evidencia en la figura 2.

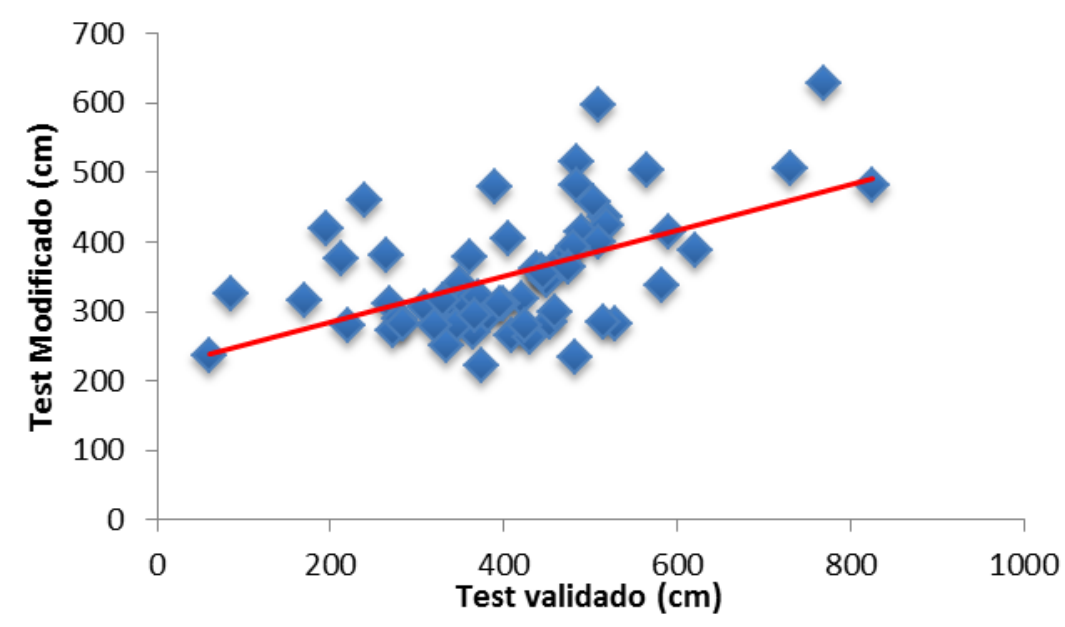

Figura 2. Correlación entre el Test Modificado de Lanzamiento del Balón Medicinal y el Test validado de Lanzamiento del Balón Medicinal, en estudiantes de primaria, $r=.533, p<.001$.

La validación se realizó también según género, en la Tabla 2 se muestra la estadística descriptiva en la relación entre el nuevo test y el test ya validado para hombres y mujeres. En las figuras $\underline{3}$ y $\underline{4}$ se muestra la relación entre estas variables para los hombres y para las mujeres, respectivamente.

\section{Tabla 2}

Estadística descriptiva en la relación entre el Test Modificado de Lanzamiento del Balón Medicinal y el Test validado de Lanzamiento del Balón Medicinal, según sexo, en estudiantes de primaria

\begin{tabular}{clc}
\hline & \multicolumn{1}{c}{ Variable } & M \\
\hline Hombres & Test Modificado & $441.45(152.64)$ \\
$(n=31)$ & Test Validado & $359.42(94.44)$ \\
Mujeres & Test Modificado & $380.88(122.52)$ \\
$(n=34)$ & Test Validado & $348.12(79.14)$ \\
\hline
\end{tabular}

Nota: se presentan valores promedio (desviaciones estándar) de las distancias obtenidas en cada una de las pruebas, los datos se encuentran en centímetros $(\mathrm{cm})$.

Para ambos sexos al realizar el análisis estadístico de correlación de Pearson (r), se concluye que sí existe una correlación directa entre el test modificado de lanzamiento del balón 
medicinal y el test validado de lanzamiento del balón medicinal, en hombres $(r=.601$; $p<.001)$ y mujeres $(r=.441 ; p=.009)$ (Figura $\underline{3}$ y $\underline{4})$.

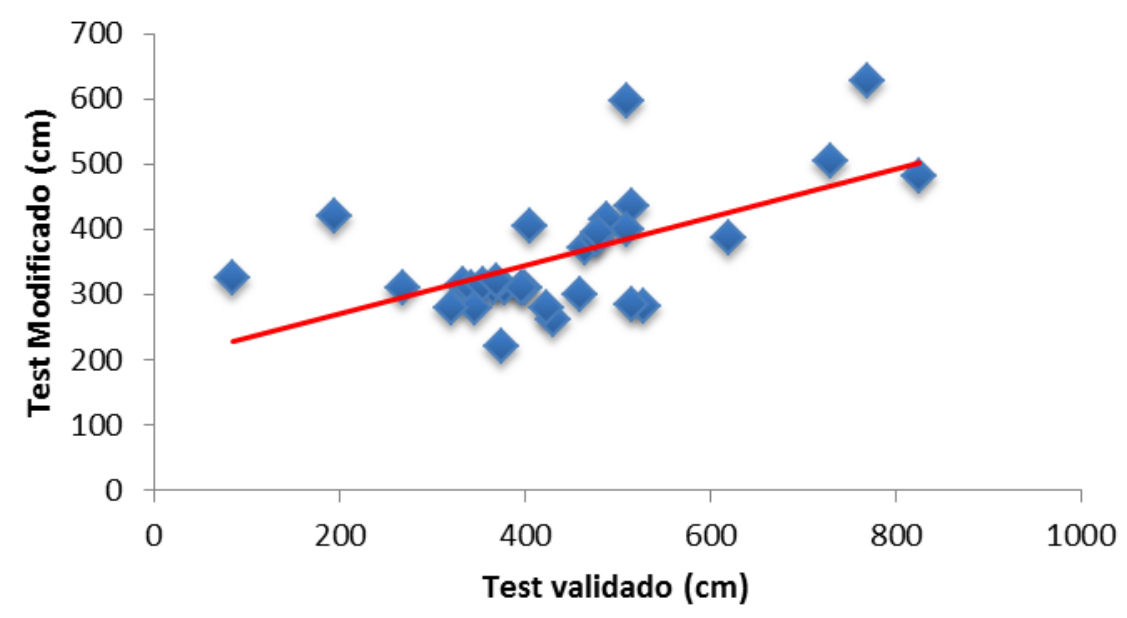

Figura 3. Correlación entre el Test Modificado de Lanzamiento del Balón Medicinal y el Test validado de Lanzamiento del Balón Medicinal, en estudiantes de primaria masculinos, $r=.601$, $\mathrm{p}<.001$.

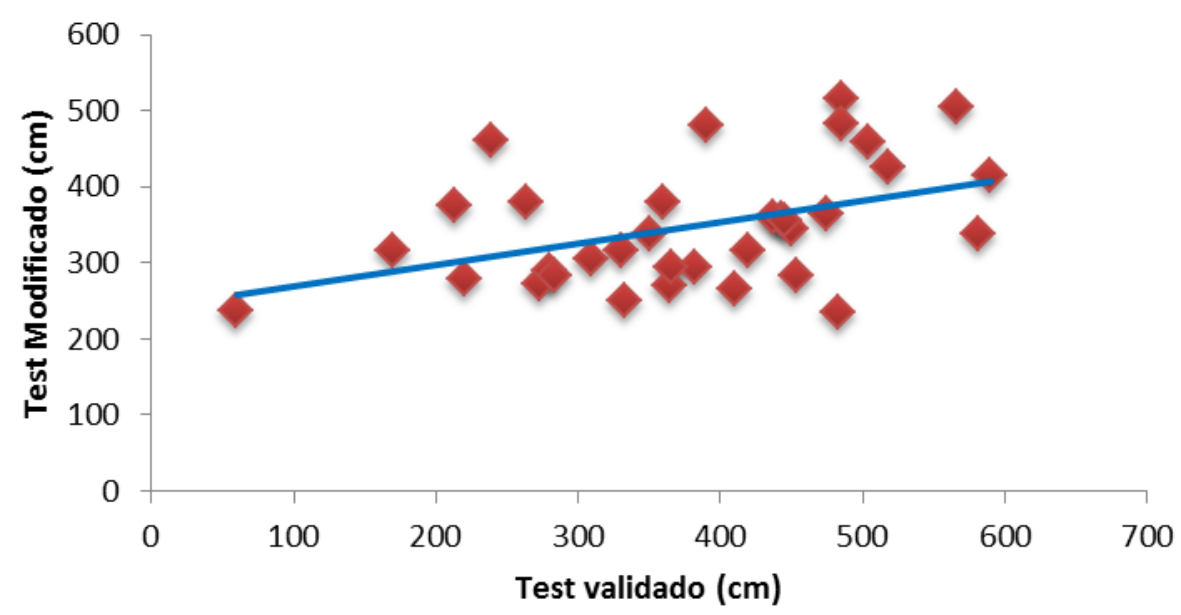

Figura 4. Correlación directa entre el Test Modificado de Lanzamiento del Balón Medicinal y el Test validado de Lanzamiento del Balón Medicinal, en estudiantes de primaria femenino, $r=.441, p=.009$.

Una vez obtenidos los datos de validez del test modificado de lanzamiento del balón medicinal, se procede a obtener los datos de confiabilidad del mismo, mediante un protocolo de test - retest; en la Tabla 3 se muestra la estadística descriptiva de la prueba para el test modificado de lanzamiento del balón medicinal. En la figura 5 se muestra la relación entre ambas ejecuciones de la prueba modificada. 


\section{Tabla 3}

Estadística descriptiva en la prueba test - retest para el Test Modificado de Lanzamiento del Balón Medicinal, en estudiantes de primaria

\begin{tabular}{cc}
\hline \multicolumn{1}{c}{ Variable } & $\mathrm{M}$ \\
\hline Test Modificado 1 & $409.51(140.02)$ \\
Test Modificado 2 & $440.63(133.40)$ \\
\hline
\end{tabular}

Nota: se presentan valores promedio (desviaciones estándar) de las distancias obtenidas en cada una de las pruebas, los datos se encuentran en centímetros $(\mathrm{cm})$. La cantidad de participantes fue de 65.

Con los datos estadísticos obtenidos de la correlación de Pearson (r), se concluye que sí existe una correlación directa en la aplicación del protocolo test - retest para el test modificado de lanzamiento del balón medicinal $(r=.454 p=.007)$ (Figura 5).

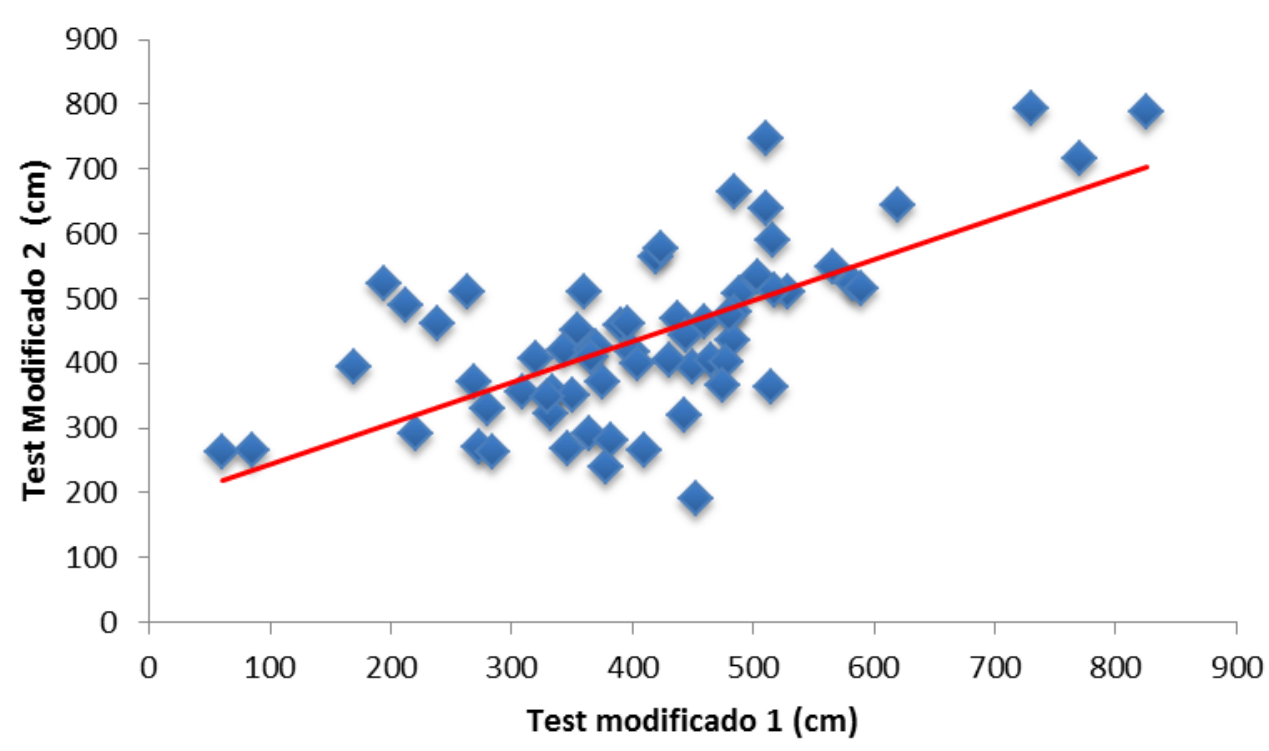

Figura 5. Correlación entre el Test Modificado de Lanzamiento del Balón Medicinal medición 1 y el Test Modificado de Lanzamiento del Balón Medicinal medición 2, en un protocolo test - retest, en estudiantes de primaria, $r=.454, p=.007$.

De igual forma la confiabilidad se determinó para cada uno de los sexos; en la Tabla 4 se muestra la estadística descriptiva en la relación entre la medición 1 del test modificado de lanzamiento del balón medicinal y la medición 2 del mismo test en hombres y mujeres. En las 
figuras $\underline{6}$ y $\underline{7}$ se muestra la relación entre el primero y segundo test para los hombres y las mujeres, respectivamente.

\section{Tabla 4}

Estadística descriptiva en la relación entre el Test Modificado de Lanzamiento del Balón Medicinal en la primera y segunda medición, según sexo, en estudiantes de primaria

\begin{tabular}{ccc}
\hline & Variable & M \\
\hline Hombres $(n=31)$ & Test Modificado 1 & $441.45(152.64)$ \\
& Test Modificado 2 & $478.10(146.66)$ \\
Mujeres & Test Modificado 1 & 380.88 (122.52) \\
$(n=34)$ & Test Modificado 2 & $406.47(111.52)$ \\
\hline \multicolumn{2}{c}{$M=$ Media; (Desviación estándar) }
\end{tabular}

Nota: se presentan valores promedio (desviaciones estándar) de las distancias obtenidas en cada una de las pruebas, los datos se encuentran en centímetros $(\mathrm{cm})$.

Al realizar el análisis estadístico de correlación de Pearson ( $r$ ) según el género, se concluye que sí existe una correlación directa entre la medición 1 del test modificado de lanzamiento del balón medicinal y la medición 2 , en hombres $(r=.769 ; p<.001)$ y mujeres $(r=.454 ; p=.007)$ (Figura $\underline{6}$ y $\underline{7})$.

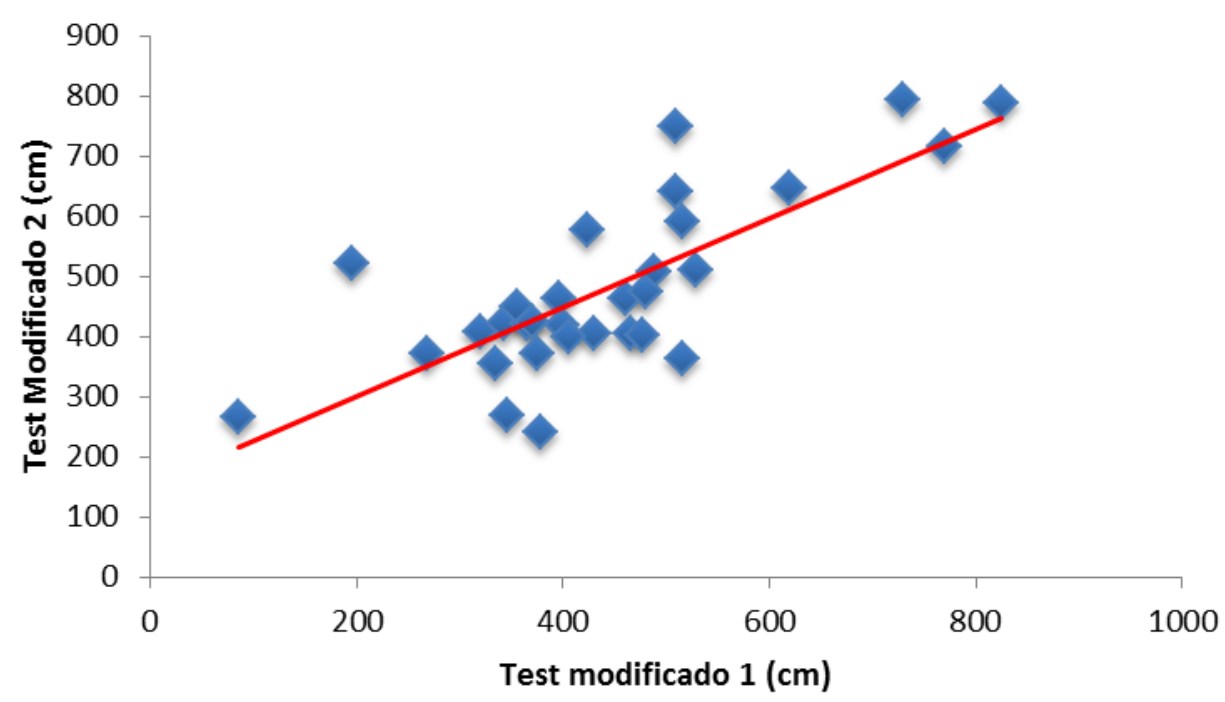

Figura 6. Correlación entre la primera medición del Test Modificado de Lanzamiento del Balón Medicinal y la segunda medición, en estudiantes de primaria masculinos, $r=.769, p<.001$. 


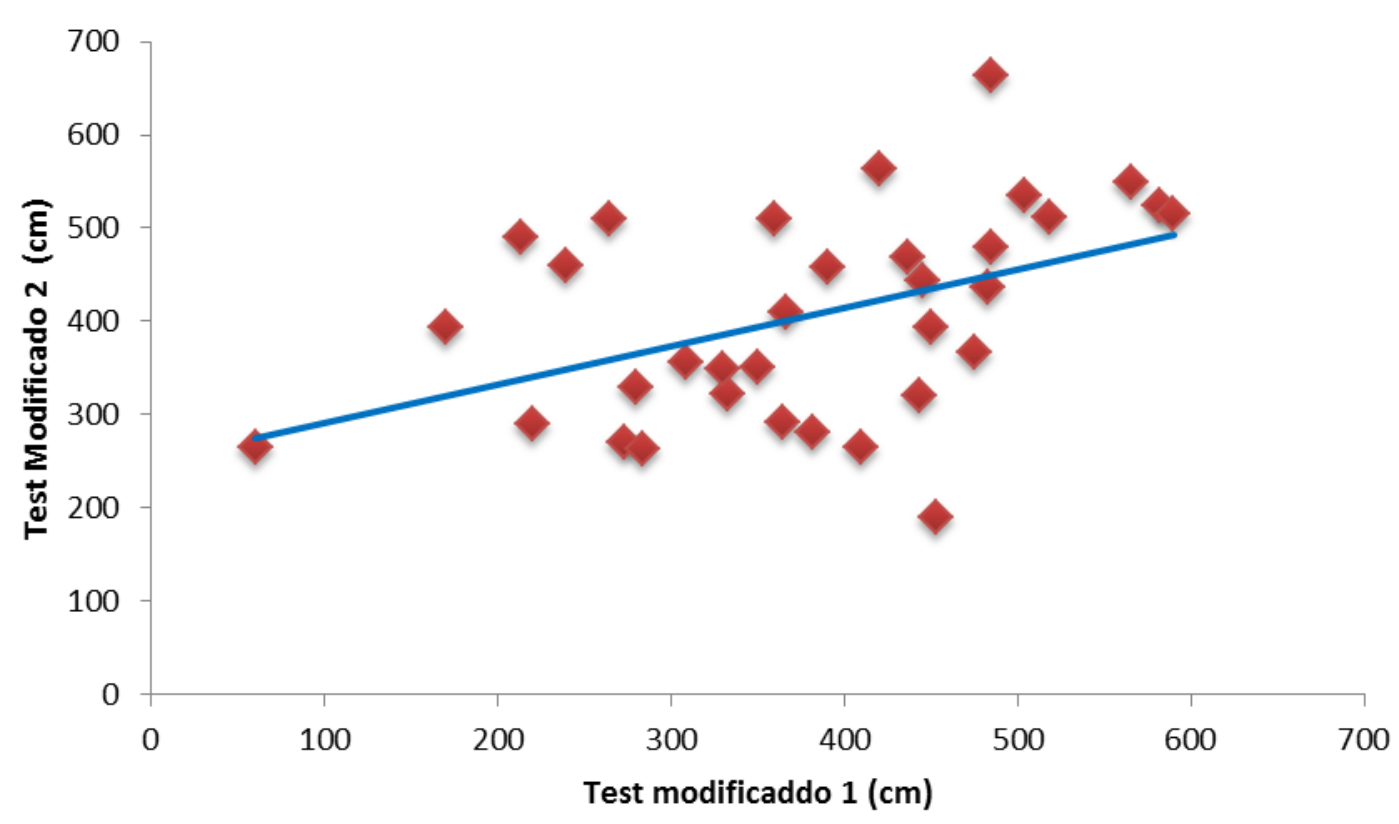

Figura 7. Correlación entre la primera medición del Test Modificado de Lanzamiento del Balón Medicinal y la segunda medición, en estudiantes de primaria femenino, $r=.454, p=.007$.

\section{Discusión}

La halterofilia es un deporte individual, acíclico debido a que la fase final del movimiento técnico no marca el inicio de una nueva repetición; el gesto técnico es de poca duración con alto gasto energético y en condiciones anaeróbicas, de máxima intensidad, con gran demanda de condición física, donde los factores principales de preparación son la fuerza y la velocidad (Cuervo y González, 1990), elementos presentes en la modificación del test de lanzamiento del balón medicinal de $2 \mathrm{~kg}$. Esto, en combinación con la similitud con el gesto técnico de los deportistas, le da al test modificado una validez aparente, conforme se ilustra en los párrafos siguientes y las figuras $\underline{4}, \underline{5}$ y $\underline{6}$.

En otros deportes los ejercicios de halterofilia son tomados como parte de su preparación, ya que la mayoría de los deportistas manifiestan adaptaciones en respuesta al entrenamiento con pesas de una manera muy avanzada y eficaz; la respuesta es más rápida, coordinada y de mayor complejidad que al utilizar otros entrenamientos de fuerza (Ortiz et al, 1996). Los estímulos de un entrenamiento de halterofilia son captados inmediatamente por los receptores, los cuales envían la información a través de las vías de conducción (nervios) hacia los centros nerviosos (médula, cerebro) en donde se elabora una respuesta a estos potentes estímulos (Wilmore y Costill, 2007), por ende la necesidad de ejecutar tests semejantes al gesto técnico que los deportistas realizan.

Para un adecuado inicio de la técnica del arranque, el levantador debe ubicar su cuerpo de la forma más simétrica posible con relación a la barra, evitando desproporciones de fuerzas que repercutan en su levantamiento; para ello el agarre debe ser simétrico sobre la longitud de la barra, cada levantador deberá con los entrenamientos establecer la distancia idónea de su agarre según sus características anatómicas. Los pies del levantador se colocarán por debajo 
de la barra y las piernas estarán entre los brazos inclinadas hacia adelante muy cerca de la palanqueta; los brazos se encuentran extendidos, hombros atrasados y la espalda recta con una tendencia a la hiperextensión. La cabeza del levantador debe mantener la línea que trae la espalda, mientras que la mirada se enfoca al frente y arriba. Una vez ubicado el levantador de esta forma estará listo para el inicio del arranque (Galván, 2008).
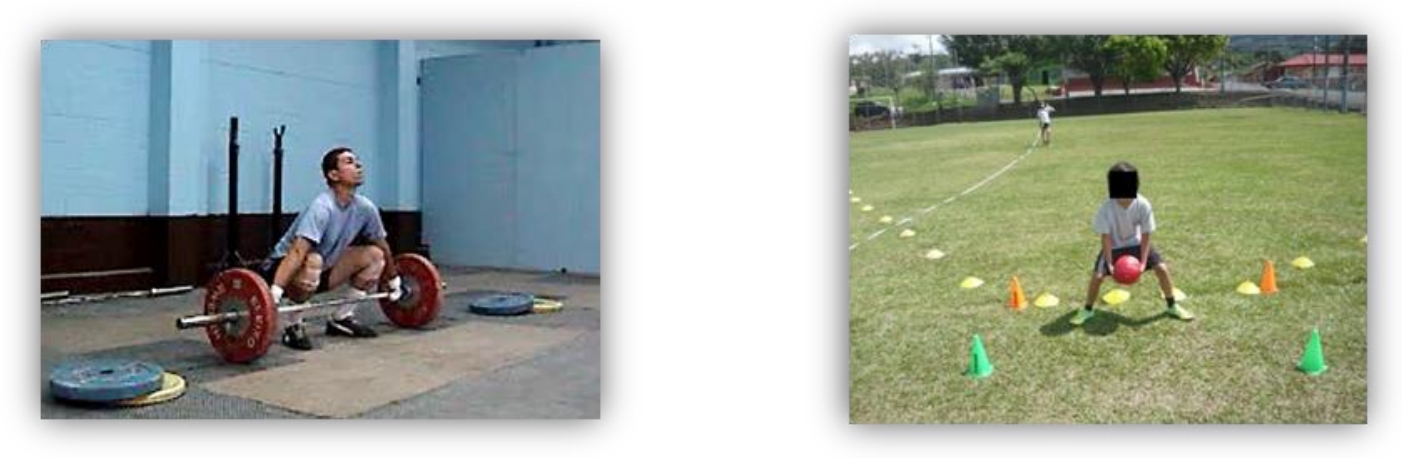

Figura 4. Comparación de la posición inicial del arranque con la posición inicial del Test Modificado de Lanzamiento del Balón Medicinal de 2 kg.

La primera parte de la técnica es la primera fase del tirón, en el cual se perderá la posición inicial con un movimiento corto y rápido para despegar la barra de la tarima; para ello se eleva la cadera, extendiendo las rodillas y retrasándolas, mientras que los músculos de la espalda y los hombros dan soporte al peso, la barra pasa muy cerca de la pierna del levantador, los hombros quedan por delante de la barra, la cabeza y el cuello en hiperextensión, los brazos aún se encuentran extendidos, llevando la barra hasta por encima de las rodillas donde finaliza el primer tirón (Galván, 2008).
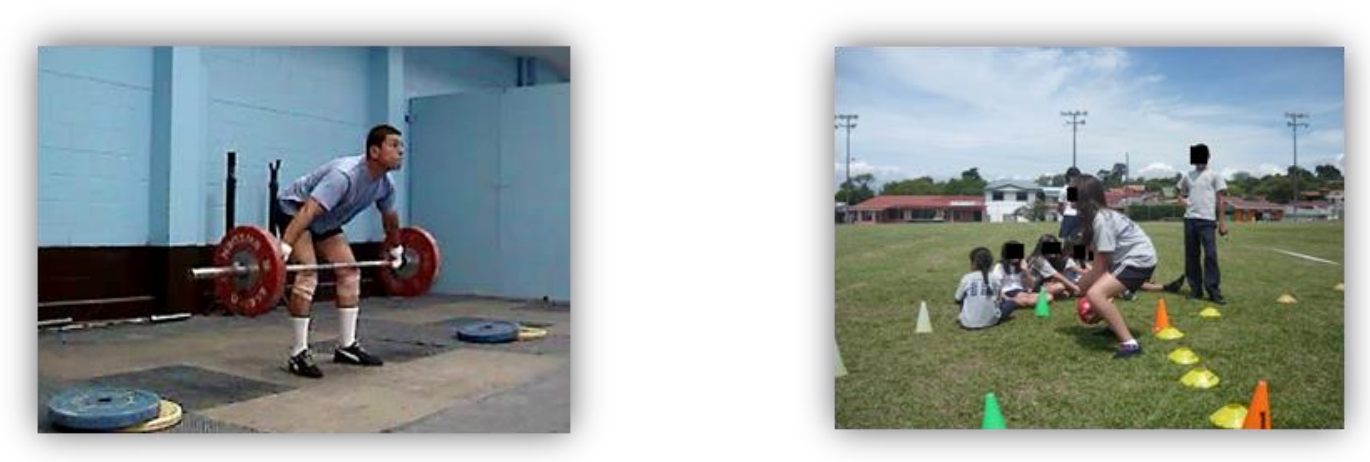

Figura 5. Comparación de la primera fase del tirón del arranque con la técnica de ejecución del Test Modificado de Lanzamiento del Balón Medicinal de $2 \mathrm{Kg}$.

De forma inmediata al primer tirón se continua con la segunda fase del tirón, el cual consiste en llevar la barra al punto de mayor altura posible; en esta fase existe un trabajo 
potente de los músculos de la espalda para generar una hiperextensión del tronco; la rodillas se flexiona, adelantándose y metiéndose por debajo de la barra, la cual pasa muy cercana al cuerpo, acercando el centro de gravedad de la barra y del levantador (Galván, 2008).
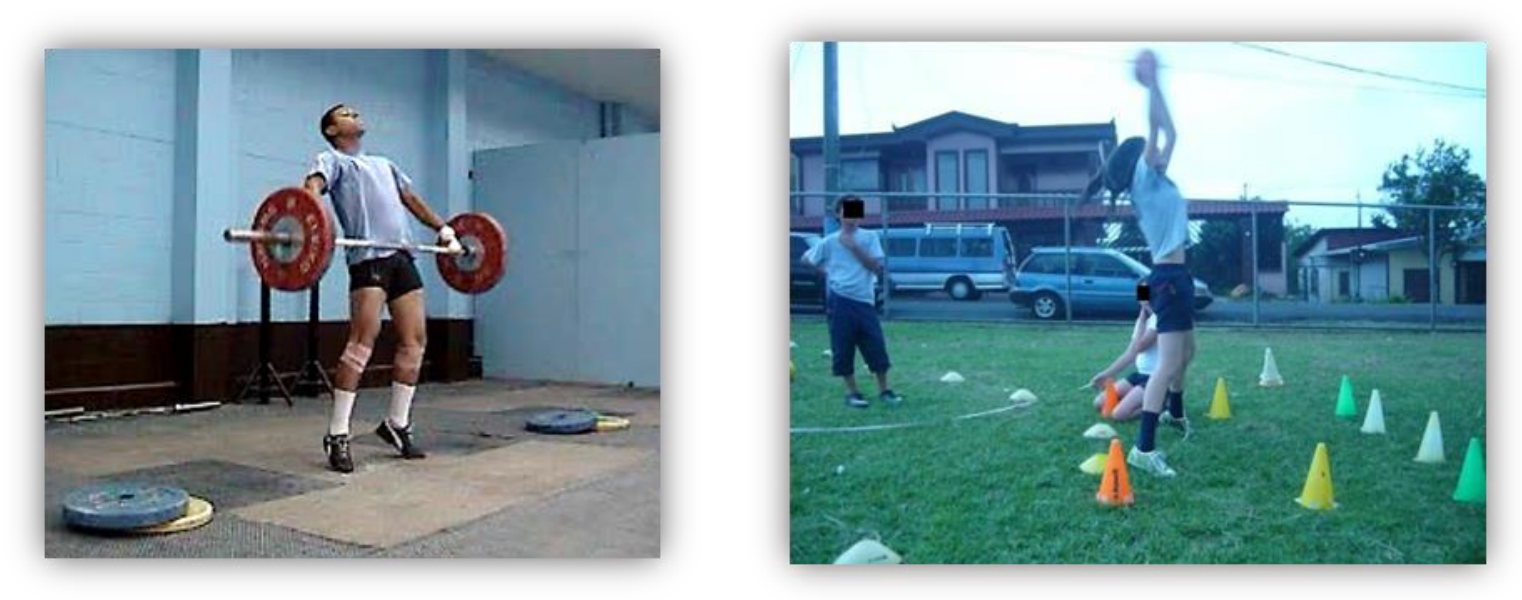

Figura 6. Comparación de la segunda fase del tirón del arranque con la técnica de ejecución del Test Modificado de Lanzamiento del Balón Medicinal de $2 \mathrm{Kg}$.

Entonces, se pretende alcanzar una extensión muy potente del cuerpo que logre elevar la barra al máximo posible, mientras que los brazos y la espalda continúan rectos, la cadera y las piernas se extienden rápidamente; el tronco desarrolla un movimiento de hiperextensión hacia arriba y hacia atrás, mientras que las caderas se moviliza hacia adelante y hacia arriba; se elevan los talones, los codos se inician a flexionar y los hombros se elevan para tirar aún más la barra hacia arriba, en una trayectoria siempre cercana al cuerpo. El segundo tirón finaliza cuando las rodillas llegan a estar en un ángulo de $160^{\circ}$ a $170^{\circ}$ y la cadera llega aproximadamente a los $170^{\circ}$.

Por último, se encuentra la fase de entrada; esta fase consiste en aprovechar la inercia de la barra en su elevación y descender debajo de ella. Una vez que la barra alcanzó su máxima altura el levantador llevará su cuerpo a introducirse por debajo de la barra, para ello despegará los pies de la tarima y los separará un poco, continúa la trayectoria de la barra con los brazos para ubicarla por encima de la cabeza, un poco retrasada, con los hombros hacia atrás y extenderá los brazos, la cadera y las rodillas se flexionan, quedando en una posición sentada, mientras que la espalda y la cabeza se mantienen rectos; la barra quedará en línea con el centro de gravedad del levantador. Una vez estable, el levantador iniciará a incorporase a la posición de pie.

Ahora bien, ¿por qué se propone el test modificado de lanzamiento del balón medicinal en la búsqueda de talentos deportivos en la halterofilia? Esto responde a dos inquietudes: la primera de ellas, contar con un elemento práctico y masivo, que permita detectar aquellos niños y niñas que presentan habilidades naturales ante un ejercicio muy similar a la ejecución técnica de este deporte, no solo por su similitud técnica, sino además por sus características 
bioenergéticas similares al gesto técnico. Bompa (2007, p.331) hace referencia a la utilización de los balones medicinales como un medio de desarrollo de la fuerza o bien, como modo de evaluación de dicha capacidad. El realizar evaluaciones de fuerza de las extremidades superiores, a través del lanzamiento del balón medicinal, se ha evidenciado que es válido y fiable (Herman y Smith, 2008), muy relacionado con los resultados obtenidos en este estudio.

Otro aspecto fundamental en la aplicación del test modificado es que permite valorar un aspecto tan delicado como lo es la fuerza en los niños. Un protocolo como el propuesto permite a través de una actividad sencilla, casi un juego, determinar quién presenta características importantes en este caso de fuerza explosiva, gracias a una buena cantidad de fibras rápidas de manera innata, sin someter al niño a un riesgoso protocolo donde se involucre el levantar algún tipo de haltera que en sí contenga una carga. Esta es la razón por la cual se determinó como peso del balón medicinal el de $2 \mathrm{~kg}$ y no un peso superior, esto a pesar que existen protocolos del mismo test con pesos superiores, pero el peso de $2 \mathrm{~kg}$ es un estímulo adecuado para estas edades y accesible a todos los practicantes del test.

Muchos investigadores y muchos médicos no han utilizado test basados en 1RM para evaluar la evolución en la fuerza muscular producida por el entrenamiento, pues parten del supuesto de que cargas de intensidad alta podrían causar daños estructurales en los niños (Frölich et al., 2005).

La prueba analizada en este estudio tiene muchas facilidades para ser aplicada en la clase de educación física, donde el docente tiene muchos estudiantes con características muy diferentes y poco tiempo para poder desarrollar habilidades específicas, pero sí puede ser un filtro para determinar y orientar a los estudiantes en relación con sus habilidades deportivas. Las clases de educación física no permiten que los niños puedan entrenarse con las cargas de entrenamiento correspondientes, por lo que un niño talentoso no tendría el estímulo adecuado para desarrollar una aptitud favorable para el deporte; es por esta razón que un docente de educación física puede aplicar este test y verificar el potencial de un niño hacia este deporte y enviarlo al club de halterofilia más próximo.

Gallahue (1982) indica que los niños antes de ingresar a un periodo de aprendizaje de las destrezas motoras especificas (11 a 13 años), debieron pasar por una serie de etapas donde se desarrollan aspectos tan importantes como los movimientos rudimentarios ( 0 a 2 años), los patrones básicos de movimiento (2 a 7 años) y las destrezas motoras generales (8 a 10 años), efectuar pruebas de talentos es la posibilidad de corregir problemas motores, problemas de coordinación, vicios en la postura corporal y otros que podrían disminuir las posibilidades de éxito de un futuro levantador de pesas.

Cuando se habla de selección de talentos deportivos no se pueden obviar las diferentes tendencias con relación a esta temática; una de ellas es el Sistema Piramidal (Sánchez, 2003), que se basa en tener muchos practicantes en la base y a través de un proceso de selección y eliminación progresivo a lo largo de los diferentes niveles, lo cual ocasiona que lleguen pocos a la cúspide. 
Otro enfoque es el selectivo - intensivo, un planteamiento avanzado con respecto a la organización del sistema para el desarrollo de los deportistas de alto nivel, que se basa en una selección más intensiva en comparación al sistema piramidal. La elección de talentos deportivos se da en contra de una acción generalizada, esta escogencia lleva hacia una selección temprana de los talentos deportivos con lo que el sistema gana en eficiencia al enfocar hacia un colectivo más pequeño y más dotado; por otra parte no se expone a una gran cantidad de individuos a esfuerzos y frustraciones innecesarias (Sánchez, 2003). Un test como el validado en este estudio permite maximizar los recursos y orientar en mejor forma el proceso. Esta selección temprana será más eficiente cuanto más numeroso sea el grupo del cual sea hecha; esto nos lleva a un modelo selectivo intensivo. Un proceso selectivo oportuno lleva a una concentración de los recursos, a una planificación a largo plazo con una dedicación prioritaria al entrenamiento, invirtiendo el tiempo necesario para lograr grandes resultados (Sánchez, 2003).

Es preciso reflexionar sobre los valores obtenidos al ejecutar como diseño estadístico la correlación de Pearson ( $r$ ) y obtener un coeficiente de correlación, el cual evalúa cómo se relacionan los puntajes de los diferentes momentos, en términos de asociación lineal (Sánchez y Echeverry, 2004). Sin embargo, la correlación no implica causalidad, la causalidad es un juicio de valor que requiere más información que un simple valor cuantitativo de un coeficiente de correlación (Fernández y Díaz, s.f.). Por consiguiente, el test modificado de lanzamiento del balón medicinal es una herramienta que el entrenador o educador físico puede utilizar, pero no como único aspecto, debe fundamentarse de otras fuentes para la toma final de las decisiones.

Con estos resultados de validez $(r=.533 ; p<.001)$ y confiabilidad $(r=.454 ; p=.007)$, la correlación al nivel de .01 (bilateral) es significativa, para Martínez (2002) y McMillan y Schumacher (2005) los coeficientes de correlación representan una relación baja o dudosa y muestra una relación positiva moderada; por ende, para ambos autores la correlación que existe no es tan fuerte; los valores de correlación deben estar preferiblemente por encima de .8. Sánchez y Echeverry (2004) indican que el hallazgo de valores altos de correlación en un proceso de validación debe interpretarse con cautela; esta misma situación con los coeficientes de correlación se evidencia en las correlaciones efectuadas para ambos sexos.

Márquez y Fernández (2012) consideran que erróneamente, se ha venido utilizando el coeficiente de correlación lineal de Pearson $(r)$ en otras investigaciones, sin tener en cuenta que expresa no el acuerdo, sino la posible presencia de una relación lineal entre los registros de dos variables; estos autores expresan que si dos instrumentos miden sistemáticamente cantidades diferentes uno del otro, la correlación será perfecta $(r=1)$, pero la concordancia nula.

A pesar de ello, la cuantificación de la fuerza de la relación lineal entre dos variables cuantitativas, se estudia por medio del cálculo del coeficiente de correlación de Pearson (Fernández y Díaz, s.f.) y se obtienen datos muy certeros con lo que respecta a la validación de un test como el aquí analizado. Los coeficientes de correlación obtenidos afirman el hecho de que el test presenta una validez idónea para los efectos por el cual fue constituido, permitiendo así visualizar la medición de la fuerza explosiva en los niños, que a su vez el entrenador podrá interpretar como un indicador de un potencial talento deportivo. 


\section{Conclusiones}

El test modificado de lanzamiento del balón medicinal de $2 \mathrm{~kg}$ mide lo que tiene que medir según los análisis estadísticos aplicados, por ende, la prueba es válida y permite ser utilizada como una herramienta para medir la fuerza explosiva de niños y niñas de 10 a 12 años; resultados que pueden tomarse en cuenta en conjunto con otra serie de variables para la detección del talento deportivo en el deporte de la halterofilia.

Es fundamental aclarar que el test modificado no predice el rendimiento que pueda obtener un niño en halterofilia. Más bien, este estudio realizó una validación indirecta, al comparar con una prueba previamente validada y obtener una validez moderada entre ambas. Esto permite a los entrenadores contar con una herramienta confiable y validada en sus procesos de selección deportiva; sin embargo, es necesaria una continuación a este estudio para poder determinar si esta prueba (o alguna otra) permitiría verificar el valor predictivo de rendimiento, lo cual sería un gran avance en el trabajo de nuevos valores deportivos. Para ello, sería necesario validar la prueba contra un criterio de oro, una referencia más clara de rendimiento que los resultados de otra prueba predictiva.

De igual forma el test modificado de lanzamiento del balón medicinal de $2 \mathrm{~kg}$ presenta coeficientes de confiabilidad significativos en el test - retest, por tal motivo se concluye que la prueba es confiable y puede ser aplicada en niños y niñas de 10 a 12 años. A pesar de que tanto los coeficientes de correlación para la validación y la confiabilidad de la prueba son significativos, los coeficientes de correlación presentan una relación baja a moderada, lo que nos indica que el test modificado de lanzamiento del balón medicinal de $2 \mathrm{~kg}$ no puede ser el único parámetro para medir la fuerza explosiva en niños y niñas de 10 a 12 años, sino que esta herramienta debe complementarse con otras que permitan de forma integral tomar las mejores decisiones.

Este tipo de trabajo abre camino para desarrollar más estudios de investigación en el desarrollo de técnicas evaluativas más precisas y especificas al gesto técnico, que puedan predecir el rendimiento de los levantadores de pesas en la técnica de arranque; como puede ser el caso de aplicar este mismo test pero no una medición como la efectuada en este estudio, sino con una medición de la altura que toma el balón medicinal o bien la combinación de ambas, altura y distancia.

La adecuada selección de los talentos deportivos en la halterofilia permite a los entrenadores maximizar los esfuerzos y los recursos, en la búsqueda de los mejores resultados deportivos; un deporte como este se debe desarrollar a través de los más diversos métodos, elaborados con base en los principios y leyes biológicas que desarrollen los diferentes aspectos de la preparación de sus deportistas.

\section{Referencias}

Aján, T. y Baroga, L. (1988). Weightlifting: fitness for all sports. Budapest: International Weightlifting Federation. (Ir a libro)

Alba, A. (1996). Test de evaluación funcional en el deporte. Armenia, Colombia: Kinesis. (Ir a libro) 
Amenabar, B. y Arruza, J. (2002). Aspectos teóricos de la iniciación deportiva. En J., Arruza (Ed), Nuevas perspectivas acerca del deporte educativo. (93-110). España: Universidad del país Vasco. (Ir a libro)

Bompa, T. (2007). Periodización. Teoría y metodología del entrenamiento. Barcelona, España: Hispano Europea. (Ir a libro)

Cuervo, P. y González, A. (1990). Levantamiento de Pesas. Deporte de Fuerza. La Habana, Cuba: Pueblo y Educación.

Fernández, S. y Díaz, S. (s.f.). Relación entre variables cuantitaivas. Recuperado de http://www.fisterra.com/mbe/investiga/var cuantitativas/var cuantitativas2.pdf

Frölich, M., Pieter, A., Giessing, J., Klein, M., Strack, A. Folder, H....Schmidtbleicher, D. (2005). Entrenamiento de la fuerza de niños y adolescentes: estado actual de la cuestión. Recuperado de: http://www.swi-uni-saarland.de/files/file/RED 241 pp5-16.pdf

Galván, M. (2008). Técnica de los ejercicios. Fedehalter. Revista digital de la Federación Española de Halterofilia, (3) 4; Recuperado de http://www.fedehalter.org/revistas/halter digital/halter digital.pdf

Gallahue, D. (1982). Understanding Motor Development in Children. New York: John Wiley \& Sons.

Guio, F. (2007). Medición de las capacidades físicas en escolares bogotanos aplicable en espacios y condiciones limitadas. Educación física y deporte, 26 (1), 35-43. Recuperado de

http=//aprendeenlinea.udea.edu.co/revistas/index.php/educacionfisicaydeporte/article/vie w/225/155

Herman, S. y Smith, D. (july, 2008). Four-week dynamic stretching warm-up intervention elicits longer-term performance benefits. The Journal of Strength and Conditioning Research, 22 (4), 1286-1297. doi: 10.1519/JSC.0b013e318173da50

Márquez, F. y Fernández, J. (2012). Evaluación de la fuerza del tren superior con plataforma de contacto. Revista Internacional de Medicina y Ciencias de la Actividad Física y el Deporte, 12 (45), 35-51. Recuperado de: http://cdeporte.rediris.es/revista/revista45/artevaluacion261.htm

Martínez, E. (2002). Pruebas de aptitud física. España, Barcelona: Paidotribo. (Ir a libro)

McMillan, J. y Schumacher, S. (2005). Investigación educativa (5 $5^{\text {ta }}$ ed.). Madrid: Pearson Educación S.A. (Ir a libro)

Ortiz, V., Gue, N., Navarro, J., Poletaev, P. y Rausell, L. (1999). Entrenamiento de fuerza y explosividad para la actividad física y el deporte de competición ( $2^{\mathrm{a}}$ ed.). España: INDE Publicaciones. (Ir a libro)

Pastor, D. (january, 2007). El entrenamiento de la fuerza en niños y jóvenes. Aplicación al rendimiento deportivo. Journal of Human Sport and Exercise, 2(1), 1-9. Recuperado de file://C:/Users/andrea/Desktop/9-137-1-PB.html

Sánchez, R. y Echeverry, J. (noviembre, 2004). Validación de Escalas de Medición en Salud. Revista de Salud Pública. 6 (3), 302-318. doi: org/10.1590/S0124-00642004000300006

Sánchez, F. (2005). Lliçó inaugural del Curs Acadèmic 2005-2006. Recuperado de: http://www.inefc.cat/inefc/AppPHP/imatges_continguts/lisonsinaugurals/Lli\%C3\%A7\%C3\%B3-05-06\%20(2).pdf

Wilmore, J. y Costill, D. (2007). Fisiología del Esfuerzo y del Deporte (6 ${ }^{\text {ta }}$ ed). Barcelona, España: Editorial Paidotribo. (Ir a libro)

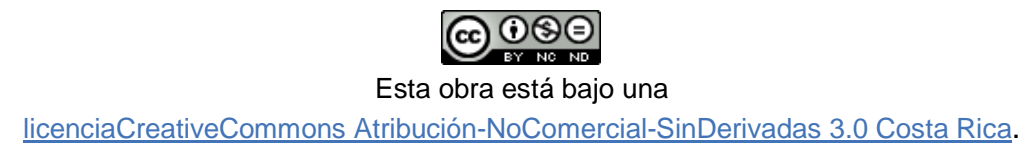

